\title{
CONVERGENCE RATE OF KRASULINA ESTIMATOR
}

\author{
JIANGNING CHEN
}

\begin{abstract}
Principal component analysis (PCA) is one of the most commonly used statistical procedures with a wide range of applications. Consider the points $X_{1}, X_{2}, \ldots, X_{n}$ are vectors drawn i.i.d. from a distribution with mean zero and covariance $\Sigma$, where $\Sigma$ is unknown. Let $A_{n}=X_{n} X_{n}^{T}$, then $E\left[A_{n}\right]=\Sigma$. This paper considers the problem of finding the smallest eigenvalue and eigenvector of matrix $\Sigma$. A classical estimator of this type is due to Krasulina 11. We are going to state the convergence proof of Krasulina for the smallest eigenvalue and corresponding eigenvector, and then find their convergence rate.
\end{abstract}

\section{INTRODUCTION}

Principal component analysis (PCA) is one of the most widely used dimension reduction techniques in data analysis. Suppose $X_{1}, X_{2}, \ldots, X_{n}$ are vectors drawn i.i.d. from a distribution with mean zero and covariance $\Sigma$, where $\Sigma \in \mathbb{R}^{d \times d}$ is unknown. Let $A_{n}=X_{n} X_{n}^{T}$, then $E\left[A_{n}\right]=\Sigma$. We are interested in finding eigenvalues of matrix $\Sigma$ and the corresponding eigenvectors if identifiable.

This problem has been intensively studied especially in the offline setting where all the observations are available at once, see [2, 4, 5, 14, 16, 17, 19. For instance, [5] derived the sharp minimax rate of estimation of the eigenvectors for the following Frobenius risk $E\left[\left\|\Theta \Theta^{T}-\hat{\Theta} \hat{\Theta}^{T}\right\|_{F}^{2}\right]$, where $\Theta=\left[\theta_{1}, \theta_{2}, \ldots, \theta_{r}\right]$ is the matrix of eigenvectors and $\hat{\Theta}$ is the corresponding estimator. Recently, [8, 9, 10, derived subtle results about the behavior of the standard PCA method in an infinite-dimensional setting.

In the high dimensional setting and for massive data sets, the computational complexity of PCA may become an issue. Indeed, for data in $\mathbb{R}^{d}$, the default method needs storage space in the order of $O\left(d^{2}\right)$. Therefore, it is interesting to develop online incremental schemes that only take one data point at a time to update estimators of eigenvectors and eigenvalues. The least storage consuming methods only need $O(d)$ space to compute one eigenvector.

Assume matrix $\Sigma$ has the standard decomposition:

$$
\Sigma=\sum_{j=1}^{d} \lambda_{j} \theta_{j} \otimes \theta_{j},
$$

where eigenvalues $\lambda_{j}$ 's satisfy: $\lambda_{1}<\lambda_{2} \leq \lambda_{3} \leq \ldots<\lambda_{d}$ and $\theta_{j}$ are the corresponding eigenvectors. We assume here that $\lambda_{1}<\lambda_{2}$ so that $\theta_{1}$ is identifiable up to sign. To compute the smallest eigenvalue and corresponding eigenvector, Krasulina [1]

Date: Nov.14 2017.

Key words and phrases. PCA, incremental, online updating, covariance matrix, eigenvector, rate of convergence, adaptive estimation. 
suggested the following stochastic gradient scheme. At time $n+1$, the estimate of the smallest eigenvector $V_{n+1}$ is updated as follows:

$$
V_{n+1}=V_{n}-\gamma_{n+1} \xi_{n+1},
$$

where $\left\{\gamma_{n}\right\}$ is the learning rate, typically, $\left\{\gamma_{n}\right\}$ is chosen such that

$$
\sum \gamma_{n}=\infty, \sum \gamma_{n}^{2}<\infty
$$

For example, $\gamma_{n}=\frac{c}{n}$ where $c$ is an absolute constant, in practice, we can choose $c=1$. And

$$
\begin{aligned}
\xi_{n+1} & =<X_{n+1}, V_{n}>\cdot X_{n+1}-\frac{<X_{n+1}, V_{n}>^{2}}{\left\|V_{n}\right\|^{2}} \cdot V_{n} \\
& =A_{n+1} \cdot V_{n}-\frac{<A_{n+1} V_{n}, V_{n}>}{\left\|V_{n}\right\|^{2}} \cdot V_{n} .
\end{aligned}
$$

There has been a lot of effort to compute the spectrum decomposition. Oja and Karhunen 13. suggested a method which is closely related to Krasulina's, they use the update for the leading eigenvector as follows:

$$
V_{n+1}=\frac{V_{n}+\gamma_{n+1}<X_{n+1}, V_{n}>X_{n+1}}{\left\|V_{n}+\gamma_{n+1}<X_{n+1}, V_{n}>X_{n+1}\right\|} .
$$

[11, 13] proved that these estimators converge almost surely under the assumption (1.1), (1.3) and $E\left[\left\|X_{n}\right\|^{k}\right]<\infty$ for some suitable $k$.

There are many other incremental estimators whose convergence has not been established yet. [18] introduces a candid covariance-free incremental PCA algorithm with assumption (1.1), they suggest the estimator:

$$
V_{n+1}=\frac{n-1-l}{n} V_{n-1}+\frac{1+l}{n} X_{n} X_{n}^{T} \frac{V_{n-1}}{\left\|V_{n-1}\right\|},
$$

where $l$ is called the amnesic parameter. With the presence of $l$, larger weight is given to new samples and the effect of old samples will fade out gradually. Typically, $l$ ranges from 2 to 4 . They also addressed the estimation of additional eigenvectors by first subtracting from the data its projection on the estimated eigenvectors, then applying (1.5). 1 considers PCA problem as stochastic optimization problem, it considers an unknown source distribution over $\mathbb{R}^{d}$, and would like to find the $\mathrm{k}$-dimensional subspace maximizing the variance of the distribution inside the subspace. They solve the problem by stochastic gradient descent, and suggests the updates:

$$
V_{n+1}=\mathcal{P}_{\text {orth }}\left(V_{n}+\eta_{n} X_{n} X_{n}^{T} V_{n}\right),
$$

where $\mathcal{P}_{\text {orth }}(V)$ performs a projection with respect to the spectral norm of $V V^{T}$ onto the set of $d \times d$ matrices with $k$ eigenvalues equal to 1 and the rest $0, \eta_{n}$ is the step size.

There also exist many results which analyze incremental PCA from the statistical perspective. They mainly show the asymptotic consistency of estimators under certain conditions. For example, 12 suggests a Block-Stochastic Power Method. [7] finds an upper bound in probability $1-\delta$ of alignment loss function $1-\frac{\left\langle V_{n}, \theta_{1}>^{2}\right.}{\left\|V_{n}\right\|^{2}}$ for Oja's estimator.

As for non-asymptotic result, 3] derives sub-optimal bound on the alignment $\operatorname{loss} L\left(V_{n}, \theta_{1}\right):=E\left[1-\frac{\leq V_{n}, \theta_{1}>^{2}}{\left\|V_{n}\right\|^{2}}\right]$, and [6] introduces Mini-batch Power Method. 
Krasulina states the convergence of the smallest eigenvalue and eigenvector estimators, but did not provide convergence rate. In this paper, we find the rate of convergence for both eigenvalue and eigenvector estimators of Krasulina (1.2) under a relatively mild assumption. Our analysis reveals a slower rate of convergence of eigenvalue estimator $\hat{\lambda_{1}}=\frac{\left\langle A_{n} V_{n}, V_{n}\right\rangle}{\left\|V_{n}\right\|^{2}}$ and corresponding eigenvector estimator $\hat{\theta_{1}}=\frac{V_{n}}{\left\|V_{n}\right\|}$ as compared to the offline setting for Krasulina's scheme.

Notations: for any vector $x \in \mathbb{R}^{d}$, we denote by $\|x\|$ the $l^{2}-$ norm of $x$. For the sake of simplicity, for any matrix $A,\|A\|$ will refer to the operator norm of $A$, specifically, $\|A\|=\sup _{u, v} \frac{<A u, v>}{\|u\|\|v\|}$. For series $\{x\}_{n},\{y\}_{n}, x_{n} \asymp_{p} y_{n}$ is defined as: $\forall \epsilon>0$, there exists a finite $M>0$ and a finite $N>0$, such that $P\left(\frac{1}{M}<\left|\frac{y_{n}}{x_{n}}\right|<M\right)<1-\epsilon$, $\forall n>N . y_{n} \lesssim_{p} x_{n}$ is defined as: $\forall \epsilon>0$, there exists a finite $M>0$ and a finite $N>0$, such that $P\left(\left|\frac{y_{n}}{x_{n}}\right|<M\right)<1-\epsilon$.

\section{Main Results}

We now state our main result:

Theorem 2.1. Assume $\lambda_{1}<\lambda_{2}$, (1.3) and $E\left\|A_{n}\right\|^{2}<\infty$, Set $g=\lambda_{2}-\lambda_{1}$. Then the Krasulina estimator (1.2) satisfies as $n \rightarrow \infty$,

$$
\left|\hat{\lambda_{1}}-\lambda_{1}\right| \asymp_{p} \frac{\|\Sigma\|}{\sqrt{n}} \cdot\left(\sqrt{E\left[\left\|A_{n}\right\|^{2}\right]} \bigvee\|\Sigma\|\right)
$$

and

$$
L\left(V_{n}, \theta_{1}\right) \asymp_{p} \frac{\|\Sigma\|}{g \sqrt{n}} \cdot\left(\sqrt{E\left[\left\|A_{n}\right\|^{2}\right]} \bigvee\|\Sigma\|\right) .
$$

In Particular, if we require the $X_{k}$ 's to be normal random vectors, then

$$
\left\|A_{n}\right\|=\left\|X_{n}\right\|^{2} \stackrel{d}{=} \sum_{j=1}^{d} \lambda_{j} Z_{j}^{2}
$$

where $Z_{j} \stackrel{i . i . d .}{\sim} N(0,1)$. Consequently, we get

$$
E\left[\left\|A_{n}\right\|^{2}\right]=E\left[\sum_{j=1}^{d} \lambda_{j}^{2} Z_{j}^{4}+2 \sum_{i \neq j} \lambda_{i} \lambda_{j} Z_{i}^{2} Z_{j}^{2}\right]=2 \operatorname{tr}\left(\Sigma^{2}\right)+\operatorname{tr}(\Sigma)^{2} \lesssim p \operatorname{tr}(\Sigma)^{2} .
$$

Thus we have following corollary:

Corollary 2.2. Let the Assumptions of Theorem 2.1 be satisfied. Assume in addition that $\left\{X_{k}\right\}$ are i.i.d. zero mean normal random vectors with covariance matrix $\Sigma$. We have for the Krasulina scheme (1.2) as $n \rightarrow \infty$ that

$$
\left|\hat{\lambda_{1}}-\lambda_{1}\right| \asymp_{p} \frac{\|\Sigma\| \operatorname{tr}(\Sigma)}{\sqrt{n}}
$$

and

$$
L\left(V_{n}, \theta_{1}\right) \asymp_{p} \frac{\|\Sigma\| \operatorname{tr}(\Sigma)}{g \sqrt{n}} .
$$

Note that for most of the times, we are interested in the top eigenvalue and the corresponding eigenvector. The scheme of Krasulina only computes the least eigenvalue and the corresponding eigenvector. However, our result is still useful, since by multiply with -1 in the original matrix, the least eigenvalue becomes the 
top eigenvalue. So that we can still use the scheme of Krasulina to compute the top eigenvalue with the same speed of convergence. The update formula (1.2) will then become:

$$
V_{n+1}=V_{n}+\gamma_{n+1} \xi_{n+1} .
$$

\section{Proof of the Theorem}

We first state a basic result in probability that will be used throughout the paper.

Lemma 3.1. Let $\left\{Y_{n}\right\}_{n}$ be a sequence of real-valued random variable. We assume that for all $n \geq 1, Y_{n}$ is zero mean and square integrable. Define $S_{n}=\sum_{k=1}^{n} Y_{k}$. If $\sum_{n>1} E\left[Y_{n}^{2}\right]<\infty$, then $\left\{S_{n}\right\}_{n}$ converges to a real-valued random variable in probability.

Proof. By definition, $S_{n}=\sum_{k=1}^{n} Y_{k}$, since $Y_{n}$ is square integrable:

$$
E\left[\left|S_{n+r}-S_{n}\right|^{2}\right]=E\left[\left(\sum_{i=n+1}^{n+r} Y_{i}\right)^{2}\right]=\sum_{i=n+1}^{n+r} E\left[Y_{i}^{2}\right]+\sum_{n+1 \leq i<j \leq n+r} 2 E\left[Y_{i} \cdot Y_{j}\right]
$$

Since $Y_{n}$ is zero mean, then for $i<j$ :

$$
E\left[Y_{i} \cdot Y_{j}\right]=E\left[E\left[Y_{i} \cdot Y_{j} \mid \mathcal{F}_{i}\right]\right]=E\left[Y_{i} \cdot E\left[Y_{j} \mid \mathcal{F}_{i}\right]\right]=0,
$$

plug it into (3.1), we obtain:

$$
E\left[\left|S_{n+r}-S_{n}\right|^{2}\right]=\sum_{i=n+1}^{n+r} E\left[Y_{i}^{2}\right] \leq \sum_{i>n} E\left[Y_{i}^{2}\right],
$$

this is the remainder term of a convergence series, thus $\left\{S_{n}\right\}_{n}$ is Cauchy, so $\left\{S_{n}\right\}_{n}$ converges to a real-valued random variable in $\mathcal{L}^{2}$. By Kolmogorov inequality, Lemma 3.1 follows.

Now, we start by bounding the asymptotic expectation of $\left\|V_{n}\right\|^{2}$ :

Lemma 3.2. $\lim _{n \rightarrow \infty} E\left\|V_{n}\right\|^{2}<\infty$.

Proof. First, we prove that $V_{n}$ and $\xi_{n+1}$ are orthogonal for any $n \geq 1$.

Let $W_{n}=X_{n+1}-\frac{\left\langle X_{n+1}, V_{n}>\right.}{\left\|V_{n}\right\|^{2}} \cdot V_{n}$, we have:

$$
\begin{aligned}
\xi_{n+1} & =<X_{n+1}, V_{n}>\cdot X_{n+1}-\frac{<X_{n+1}, V_{n}>^{2}}{\left\|V_{n}\right\|^{2}} \cdot V_{n} \\
& =<X_{n+1}, V_{n}>\left(X_{n+1}-\frac{<X_{n+1}, V_{n}>}{\left\|V_{n}\right\|^{2}} \cdot V_{n}\right) \\
& =<X_{n+1}, V_{n}>\cdot W_{n} .
\end{aligned}
$$

We note that $<W_{n}, V_{n}>=0$, so

$$
\left\|\xi_{n+1}\right\|=<X_{n+1}, V_{n}>\cdot\left\|W_{n}\right\| \leq<X_{n+1}, V_{n}>\cdot\left\|X_{n+1}\right\| \leq\left\|X_{n+1}\right\|^{2}\left\|V_{n}\right\|,
$$

thus:

$$
E\left[\left\|\xi_{n+1}\right\| \mid \mathcal{F}_{n}\right] \leq E\left[\left\|X_{n+1}\right\|^{2}\right] \cdot\left\|V_{n}\right\|=\operatorname{tr}(\Sigma)\left\|V_{n}\right\| .
$$

Now since $\xi_{n} \perp V_{n-1}$, we have

$$
\left\|V_{n}\right\|^{2}=\left\|V_{n-1}-\gamma_{n} \xi_{n}\right\|^{2}=\left\|V_{n-1}\right\|^{2}+\gamma_{n}^{2}\left\|\xi_{n}\right\|^{2}
$$


thus:

$$
\begin{aligned}
E\left[\left\|V_{n}\right\|^{2} \mid \mathcal{F}_{n-1}\right] & =\left\|V_{n-1}\right\|^{2}+\gamma_{n}^{2} E\left[\left\|\xi_{n}\right\|^{2} \mid \mathcal{F}_{n-1}\right] \\
& \leq\left\|V_{n-1}\right\|^{2}+\gamma_{n}^{2} \operatorname{tr}(\Sigma)^{2}\left\|V_{n-1}\right\|^{2} \\
& =\left(1+\gamma_{n}^{2} \operatorname{tr}(\Sigma)^{2}\right)\left\|V_{n-1}\right\|^{2}
\end{aligned}
$$

Thus:

$$
\begin{aligned}
E\left\|V_{n}\right\|^{2} & \leq\left(1+\gamma_{n}^{2} \operatorname{tr}(\Sigma)^{2}\right) E\left\|V_{n-1}\right\|^{2} \\
& \leq \cdots \leq \prod_{i=2}^{n}\left(1+\gamma_{i}^{2} \operatorname{tr}(\Sigma)^{2}\right) \cdot E\left\|V_{1}\right\|^{2}
\end{aligned}
$$

By assumption (1.3), we have $\sum_{i=1}^{\infty} \gamma_{i}^{2} \operatorname{tr}(\Sigma)^{2}<\infty$, thus: $\prod_{i=1}^{n-1}\left(1+\gamma_{i}^{2} \operatorname{tr}(\Sigma)^{2}\right)<$ $\infty$, thus $\lim _{n \rightarrow \infty} E\left\|V_{n}\right\|^{2}<\infty$.

Next, let $\mu\left(V_{n}\right)=\frac{\leq \Sigma V_{n}, V_{n}>}{\left\|V_{n}\right\|^{2}}$, and $a_{1}^{(n)}=<V_{n}, \theta_{1}>$. We first prove the convergence in probability of the sequence of $V_{n}$ and $a_{1}^{(n)}$. Specifically, $\mu\left(V_{n}\right)$ converges to $\lambda_{1}$, and $V_{n}$ converges to a vector which is alined with $\theta_{1}$. To prove that, we can recursively properly apply the inequality, to show the Cauchy property of sequence $\mu\left(V_{n}\right)$ and $a_{1}^{(n)}$.

Lemma 3.3. $\mu\left(V_{n}\right)=\frac{\left\langle\Sigma V_{n}, V_{n}>\right.}{\left\|V_{n}\right\|^{2}}$ converges a.s. to $\mu$ as $n \rightarrow \infty$.

Proof.

$$
\begin{aligned}
\mu\left(V_{n+1}\right)= & \frac{\left\langle\Sigma V_{n}-\gamma_{n+1} \cdot \Sigma \xi_{n+1}, V_{n}-\gamma_{n+1} \xi_{n+1}>\right.}{\left\|V_{n}-\gamma_{n+1} \xi_{n+1}\right\|^{2}} \\
= & \frac{<\Sigma V_{n}, V_{n}>+\gamma_{n+1}^{2}<\Sigma \xi_{n+1}, \xi_{n+1}>-2 \gamma_{n+1}<\xi_{n+1}, \Sigma V_{n}>}{\left\|V_{n}\right\|^{2}+\gamma_{n+1}^{2}\left\|\xi_{n+1}\right\|^{2}} \\
= & \frac{1}{1+\gamma_{n+1}^{2} \frac{\left\|\xi_{n+1}\right\|^{2}}{\left\|V_{n}\right\|^{2}}}\left(\mu\left(V_{n}\right)-2 \gamma_{n+1} \frac{<\xi_{n+1}, \Sigma V_{n}>}{\left\|V_{n}\right\|^{2}}\right. \\
& \left.+\gamma_{n+1}^{2} \frac{<\Sigma \xi_{n+1}, \xi_{n+1}>}{\left\|V_{n}\right\|^{2}}\right)
\end{aligned}
$$

Since:

$$
\begin{aligned}
<\xi_{n+1}, \Sigma V_{n}>= & <A_{n+1} V_{n}, \Sigma V_{n}>-\frac{<A_{n+1} V_{n}, V_{n}><\Sigma V_{n}, V_{n}>}{\left\|V_{n}\right\|^{2}} \\
= & \left\|\Sigma V_{n}\right\|^{2}-\frac{<\Sigma V_{n}, V_{n}>^{2}}{\left\|V_{n}\right\|^{2}}+<A_{n+1} V_{n}, \Sigma V_{n}>-\left\|\Sigma V_{n}\right\|^{2} \\
& \quad-\frac{<A_{n+1} V_{n}, V_{n}><\Sigma V_{n}, V_{n}>}{\left\|V_{n}\right\|^{2}}+\frac{<\Sigma V_{n}, V_{n}>^{2}}{\left\|V_{n}\right\|^{2}} \\
= & \left(<\left(A_{n+1}-\Sigma\right) V_{n}, \Sigma V_{n}>-\frac{<\left(A_{n+1}-\Sigma\right) V_{n}, V_{n}>}{\left\|V_{n}\right\|^{2}}\right. \\
& \left.\cdot<\Sigma V_{n}, V_{n}>\right)+\left(\left\|\Sigma V_{n}\right\|^{2}-\frac{<\Sigma V_{n}, V_{n}>^{2}}{\left\|V_{n}\right\|^{2}}\right)
\end{aligned}
$$

Let

$$
f\left(V_{n}\right)=\frac{\left\|\Sigma V_{n}\right\|^{2}}{\left\|V_{n}\right\|^{2}}-\frac{<\Sigma V_{n}, V_{n}>^{2}}{\left\|V_{n}\right\|^{4}},
$$




$$
Z_{n}=\frac{<\left(A_{n+1}-\Sigma\right) V_{n}, \Sigma V_{n}>}{\left\|V_{n}\right\|^{2}}-\frac{<\left(A_{n+1}-\Sigma\right) V_{n}, V_{n}>}{\left\|V_{n}\right\|^{4}} \cdot\left\langle\Sigma V_{n}, V_{n}>,\right.
$$

thus: $\frac{\left\langle\xi_{n+1}, \Sigma V_{n}>\right.}{\left\|V_{n}\right\|^{2}}=f\left(V_{n}\right)+Z_{n}$.

so $\mu\left(V_{n+1}\right)=\frac{1}{1+\gamma_{n+1}^{2} \frac{\left\|\xi_{n+1}\right\|^{2}}{\left\|V_{n}\right\|^{2}}}\left(\mu\left(V_{n}\right)-2 \gamma_{n+1} f\left(V_{n}\right)-2 \gamma_{n+1} Z_{n}+\gamma_{n+1}^{2} \frac{<\Sigma \xi_{n+1}, \xi_{n+1}>}{\left\|V_{n}\right\|^{2}}\right)$.

Let

$$
a_{n}=\gamma_{n+1} Z_{n}, b_{n}=\gamma_{n+1}^{2} \frac{<\Sigma \xi_{n+1}, \xi_{n+1}>}{\left\|V_{n}\right\|^{2}}, c_{n}=\frac{1}{1+\gamma_{n+1}^{2} \frac{\left\|\xi_{n+1}\right\|^{2}}{\left\|V_{n}\right\|^{2}}},
$$

thus:

$$
\mu\left(V_{n+1}\right)=c_{n} \cdot\left(\mu\left(V_{n}\right)-2 \gamma_{n+1} f\left(V_{n}\right)-2 a_{n}+b_{n}\right) .
$$

Now we have:

$$
\mu\left(V_{n+1}\right)-c_{n} \cdot \mu\left(V_{n}\right)=-2 \gamma_{n+1} c_{n} f\left(V_{n}\right)-2 a_{n} c_{n}+b_{n} c_{n} .
$$

For series $\left\{a_{n}\right\}$, since $Z_{n}$ is centered and $E\left[Z_{n}^{2}\right]$ is bounded, by lemma 3.1

$$
\sum_{i>k} \operatorname{Var}\left(a_{i}\right) \asymp_{p} \sum_{i>k} \gamma_{i}^{2}<\infty,
$$

thus $\sum_{n=1}^{\infty} a_{n}<\infty$.

For series $\left\{b_{n}\right\}$, by (3.2):

$$
E\left[\left\|\xi_{n}\right\| \mid \mathcal{F}_{n-1}\right] \leq \operatorname{tr}(\Sigma)\left\|V_{n}\right\|
$$

thus

$$
E\left[b_{n} \mid \mathcal{F}_{n}\right]=\gamma_{n+1}^{2} E\left[\frac{<\Sigma \xi_{n+1}, \xi_{n+1}>}{\left\|V_{n}\right\|^{2}} \mid \mathcal{F}_{n}\right] \leq \gamma_{n+1}^{2}\|\Sigma\| \operatorname{tr}(\Sigma)^{2} .
$$

By (3.2), we have $\sum_{n=1}^{\infty} b_{n}<\infty$.

For series $\left\{c_{n}\right\}, \prod c_{n}=\prod \frac{1}{1+\gamma_{n+1}^{2} \frac{\left\|\xi_{n+1}\right\|^{2}}{\left\|V_{n}\right\|^{2}}}$ converges when $\prod 1+\gamma_{n+1}^{2} \frac{\left\|\xi_{n+1}\right\|^{2}}{\left\|V_{n}\right\|^{2}}$ converges. $\prod 1+\gamma_{n+1}^{2} \frac{\left\|\xi_{n+1}\right\|^{2}}{\left\|V_{n}\right\|^{2}}$ has the same convergence properties as $\sum \gamma_{n+1}^{2} \frac{\left\|\xi_{n+1}\right\|^{2}}{\left\|V_{n}\right\|^{2}}$. By (3.2),

$$
E\left[\frac{\left\|\xi_{n+1}\right\|^{2}}{\left\|V_{n}\right\|^{2}} \mid \mathcal{F}_{n}\right] \leq \operatorname{tr}(\Sigma)^{2}
$$

we have $\prod_{n=1}^{\infty} c_{n}<\infty$.

And by Cauchy-Schwartz inequality:

$$
f\left(V_{n}\right)=\frac{\left\|\Sigma V_{n}\right\|^{2}}{\left\|V_{n}\right\|^{2}}-\frac{<\Sigma V_{n}, V_{n}>^{2}}{\left\|V_{n}\right\|^{4}} \geq 0
$$

Now, if $\lim \inf \mu\left(V_{n}\right)<\lim \sup \mu\left(V_{n}\right)$, choose $a, b$ such that $\liminf \mu\left(V_{n}\right)<a<$ $b<\lim \sup \mu\left(V_{n}\right)$, find $m_{1}, n_{1}$ large enough, such that $\mu\left(V_{n_{1}}\right)<a, \mu\left(V_{m_{1}}\right)>b$, and for all $n_{1}<j<m_{1}$, we have $a \leq \mu\left(V_{j}\right) \leq b$. Thus:

$$
\mu\left(V_{m_{1}}\right)-\mu\left(V_{n_{1}}\right) \prod_{i=n_{1}}^{m_{1}-1} c_{i}>b-a .
$$


On the other hand:

$$
\begin{aligned}
(3.8) \mu\left(V_{m_{1}}\right)-\mu\left(V_{n_{1}}\right) \prod_{i=n_{1}}^{m_{1}-1} c_{i} & =\sum_{j=n_{1}}^{m_{1}-1}\left[\left(-2 \gamma_{j+1} \cdot f\left(V_{j}\right)-2 a_{j}+b_{j}\right) \cdot \prod_{i=j}^{m_{1}-1} c_{j}\right] \\
\leq & \sum_{j=n_{1}}^{m_{1}-1}\left[\left(-2 a_{j}+b_{j}\right) \cdot \prod_{i=j}^{m_{1}-1} c_{j}\right] \\
& \rightarrow 0 \text { as } n_{1}, m_{1} \rightarrow \infty
\end{aligned}
$$

which is a contradiction, thus $\mu\left(V_{n}\right) \rightarrow \mu$ with probability 1 .

Lemma 3.4. $a_{1}^{(n)}=<V_{n}, \theta_{1}>$, where $\theta_{1}$ is the eigenvector of $\lambda_{1}, a_{1}^{(n)}$ converges to some value $a_{1}$ with probability 1 as $n \rightarrow \infty$.

Proof. Since $V_{n+1}=V_{n}-\gamma_{n+1} \xi_{n+1}, \xi_{n+1}=A_{n+1} V_{n}-\frac{\left\langle A_{n+1} V_{n}, V_{n}>\right.}{\left\|V_{n}\right\|^{2}} V_{n}$, by definition of $a_{1}^{(n)}=<V_{n}, \theta_{1}>$ and $\mu\left(V_{n}\right)=\frac{\left\langle\Sigma V_{n}, V_{n}>\right.}{\left\|V_{n}\right\|^{2}}$, also by the nature: $\left\langle\Sigma V_{n}, \theta_{1}\right\rangle=<$ $V_{n}, \Sigma \theta_{1}>=<V_{n}, \lambda_{1} \theta_{1}>=\lambda_{1} a_{1}^{(n)}$, we have:

$$
\begin{aligned}
a_{1}^{(n+1)}= & <V_{n+1}, \theta_{1}>=<V_{n}-\gamma_{n+1} \xi_{n+1}, \theta_{1}> \\
= & <V_{n}, \theta_{1}>-\gamma_{n+1}<A_{n+1} V_{n}-\frac{<A_{n+1} V_{n}, V_{n}>}{\left\|V_{n}\right\|^{2}} V_{n}, \theta_{1}> \\
= & a_{1}^{(n)}+\gamma_{n+1}<\frac{<\Sigma V_{n}, V_{n}>}{\left\|V_{n}\right\|^{2}} V_{n}+\frac{<\left(A_{n+1}-\Sigma\right) V_{n}, V_{n}>}{\left\|V_{n}\right\|^{2}} V_{n}-\Sigma V_{n} \\
& +\left(\Sigma-A_{n+1}\right) V_{n}, \theta_{1}> \\
= & a_{1}^{(n)}+\gamma_{n+1}\left(\mu\left(V_{n}\right)-\lambda_{1}\right) a_{1}^{(n)}+\gamma_{n+1} Z_{n}^{\prime} \\
= & a_{1}^{(n)}\left(1+\gamma_{n+1}\left(\mu\left(V_{n}\right)-\lambda_{1}\right)\right)+\gamma_{n+1} Z_{n}^{\prime},
\end{aligned}
$$

where $Z_{n}^{\prime}=<\left(\Sigma-A_{n+1}\right) V_{n}, \theta_{1}>+\frac{<\left(A_{n+1}-\Sigma\right) V_{n}, V_{n}>}{\left\|V_{n}\right\|^{2}} a_{1}^{(n)}$.

Since $E\left[\left\|V_{n}\right\|^{2}\right]=E\left[\left\|V_{n-1}\right\|^{2}\right]+\gamma_{n}^{2} E\left[\left\|\xi_{n}\right\|^{2}\right] \leq E\left[\left\|V_{n_{1}}\right\|^{2}\right]+\gamma_{n}^{2}\|\Sigma\|^{2} E\left\|V_{n-1}\right\|^{2} \leq$ $\prod_{n=1}^{\infty}\left(1+\gamma_{n}^{2}\|\Sigma\|^{2}\right) \leq \infty, Z_{n}^{\prime}$ is centered and $E\left[Z_{n}^{\prime 2}\right]$ is bounded, by lemma 3.1 . $\sum_{n=1}^{\infty} \gamma_{n} Z_{n}^{\prime}<\infty$.

Now, if $\liminf a_{1}^{(n)}<\limsup a_{1}^{(n)}$, choose $a, b$ such that $\liminf a_{1}^{(n)}<a<b<$ $\limsup a_{1}^{(n)}$, find $m_{1}, n_{1}$, such that: $m_{1} \geq n_{1} \geq N, a_{1}^{\left(m_{1}\right)}<a, a_{1}^{\left(n_{1}\right)}>b$, for $j \in\left(n_{1}, m_{1}\right), a \leq a_{1}^{(j)} \leq b$. Since $\lambda_{1}$ is the smallest eigenvalue, $\mu\left(V_{k}\right) \geq \lambda_{1}$.

Thus:

$$
a_{1}^{\left(m_{1}\right)}-a_{1}^{\left(n_{1}\right)} \prod_{k=n_{1}}^{m_{1}}\left(1+\gamma_{k+1}\left(\mu\left(V_{k}\right)-\lambda_{1}\right)\right) \leq a_{1}^{\left(m_{1}\right)}-a_{1}^{\left(n_{1}\right)}<a-b \leq 0 .
$$

On the other hand: 


$$
\begin{aligned}
& a_{1}^{\left(m_{1}\right)}-a_{1}^{\left(n_{1}\right)} \prod_{k=n_{1}}^{m_{1}}\left(1+\gamma_{k+1}\left(\mu\left(V_{k}\right)-\lambda_{1}\right)\right) \\
= & \sum_{j=n_{1}}^{m_{1}-1} \gamma_{j} Z_{j}^{\prime} \prod_{i=j}^{m-1}\left(1+\gamma_{j+1}\left(\mu\left(V_{j}\right)-\lambda_{1}\right)\right) \\
\geq & \sum_{j=n_{1}}^{m_{1}-1} \gamma_{j} Z_{j}^{\prime}
\end{aligned}
$$

Since $\sum_{j=1}^{\infty} \gamma_{j} Z_{j}^{\prime}<\infty$, let $n_{1} \rightarrow \infty$, we can let $\sum_{j=n_{1}}^{m_{1}-1} \gamma_{j} Z_{j}^{\prime}$ as closed to 0 as we want, which is a contradiction.

Thus $a_{1}^{(n)} \rightarrow a_{1}$ with probability 1 .

Now we get the idea that $\mu\left(V_{n}\right)$ and $a_{1}^{(n)}$ are both convergence with probability 1 , and by the proof above, all coefficients in (3.6) are convergence with probability 1 , so does the part $\gamma_{n+1} c_{n} f\left(V_{n}\right)$. By find the convergence rate for each of these parts, we can find the convergence rate for $\mu\left(V_{n}\right)$.

Lemma 3.5. (1) $\mu\left(V_{n}\right) \rightarrow \lambda_{1}$ as $n \rightarrow \infty$ with probability 1 , and (2) the convergence rate of $\frac{\left\langle A_{n} V_{n}, V_{n}>\right.}{\left\|V_{n}\right\|^{2}}$ to $\lambda_{1}$ is in the order of $O\left(\frac{\|\Sigma\|}{\sqrt{n}} \cdot\left(\sqrt{E\left[\left\|A_{n}\right\|^{2}\right]}\right) \bigvee\|\Sigma\|\right)$.

Proof. (1)

$$
\begin{aligned}
a_{1}^{(n+1)}= & <V_{n+1}, \theta_{1}>=<V_{n+1}, \theta_{1}>=<V_{n}-\gamma_{n+1} \xi_{n+1}, \theta_{1}> \\
= & <V_{n}, \theta_{1}>-\gamma_{n+1}<A_{n+1} V_{n}-\frac{<A_{n+1} V_{n}, V_{n}>}{\left\|V_{n}\right\|^{2}}, \theta_{1}> \\
= & a_{1}^{(n)}+\gamma_{n+1} \frac{<A_{n+1} V_{n}, V_{n}>}{\left\|V_{n}\right\|^{2}} a_{1}^{(n)}-\gamma_{n+1}<A_{n+1} V_{n}, \theta_{1}> \\
= & a_{1}^{(n)}+\gamma_{n+1} \frac{<\Sigma V_{n}, V_{n}>}{\left\|V_{n}\right\|^{2}} a_{1}^{(n)}-\gamma_{n+1}<V_{n}, \Sigma \theta_{1}> \\
& +\gamma_{n+1} \frac{<A_{n+1} V_{n}, V_{n}>}{\left\|V_{n}\right\|^{2}} a_{1}^{(n)}-\gamma_{n+1}<A_{n+1} V_{n}, \theta_{1}> \\
& -\gamma_{n+1} \frac{<\Sigma V_{n}, V_{n}>}{\left\|V_{n}\right\|^{2}} a_{1}^{(n)}+\gamma_{n+1}<V_{n}, \Sigma \theta_{1}> \\
= & a_{1}^{(n)}\left(1+\gamma_{n+1}\left(\mu\left(V_{n}\right)-\lambda_{1}\right)\right)+\gamma_{n+1} Z_{n}^{\prime},
\end{aligned}
$$

where $Z_{n}^{\prime}=<\left(\Sigma-A_{n+1}\right) V_{n}, \theta_{1}>+\frac{<\left(A_{n+1}-\Sigma\right) V_{n}, V_{n}>}{\left\|V_{n}\right\|^{2}} a_{1}^{(n)}$, which is centered and bounded, then by Jensen's inequality:

$$
\begin{aligned}
E\left|a_{1}^{(n+1)}\right| & \geq E\left|a_{1}^{(n)}\right|\left(1+\gamma_{n+1}\left(\frac{E\left[\mu\left(V_{n}\right)\left|a_{1}^{(n)}\right|\right]}{E\left|a_{1}^{(n)}\right|}-\lambda_{1}\right)\right) \\
& \geq \prod_{k=1}^{n}\left(1+\gamma_{k+1}\left(\frac{E\left[\mu\left(V_{k}\right)\left|a_{1}^{(k)}\right|\right]}{E\left|a_{1}^{(k)}\right|}-\lambda_{1}\right)\right) E\left|a_{1}^{(1)}\right|
\end{aligned}
$$

By Lemma 3.4. $\left\{a_{1}^{(n)}\right\}$ convergence, then

$$
\prod_{k=1}^{\infty}\left(1+\gamma_{k+1}\left(\frac{E\left[\mu\left(V_{k}\right)\left|a_{1}^{(k)}\right|\right]}{E\left|a_{1}^{(k)}\right|}-\lambda_{1}\right)\right)<\infty
$$


thus:

$$
\sum_{k=1}^{\infty} \gamma_{k+1}\left(\frac{E\left[\mu\left(V_{k}\right)\left|a_{1}^{(k)}\right|\right]}{E\left|a_{1}^{(k)}\right|}-\lambda_{1}\right)<\infty .
$$

By (1.3), $\lim _{k \rightarrow \infty} \frac{E\left[\mu\left(V_{k}\right)\left|a_{1}^{(k)}\right|\right]}{E\left|a_{1}^{(k)}\right|}-\lambda_{1}=0$.

By dominant convergence theorem: $\lim _{k \rightarrow \infty} a_{1}^{(k)}=a_{1}, \lim _{k \rightarrow \infty} \mu\left(V_{k}\right)=\mu$. Thus: $\frac{\mu a_{1}}{a_{1}}=\lambda_{1}$, therefore, $\mu=\lambda_{1}$.

$(2)$

$$
\begin{aligned}
\lambda_{1}-\frac{<A_{n} V_{n}, V_{n}>}{\left\|V_{n}\right\|^{2}} & =\left(\lambda_{1}-\mu\left(V_{n}\right)\right)+\left(\mu\left(V_{n}\right)-\frac{<A_{n} V_{n}, V_{n}>}{\left\|V_{n}\right\|^{2}}\right) \\
& =\left(\lambda_{1}-\mu\left(V_{n}\right)\right)+\left(\frac{<\left(\Sigma-A_{n}\right) V_{n}, V_{n}>}{\left\|V_{n}\right\|^{2}}\right)
\end{aligned}
$$

Since $E\left[\frac{<\left(\Sigma-A_{n}\right) V_{n}, V_{n}>}{\left\|V_{n}\right\|^{2}}\right]=0$, we only need to consider $\left|\lambda_{1}-\mu\left(V_{n}\right)\right|$. From (3.6) we have:

$\mu\left(V_{n+1}\right)-c_{n} \cdot \mu\left(V_{n}\right)=-2 \gamma_{n+1} c_{n} f\left(V_{n}\right)-2 a_{n} c_{n}+b_{n} c_{n}=\left(-2 \gamma_{n+1} f\left(V_{n}\right)-2 a_{n}+b_{n}\right) c_{n}$, where $a_{j}, b_{j}$ and $c_{j}$ are defined the same as (3.5). The same way as we get (3.8), keep increase $V_{n+1}$ to $V_{m}$ recursively, we have:

$$
\mu\left(V_{m}\right)-\mu\left(V_{n}\right) \prod_{i=n}^{m-1} c_{i}=\sum_{j=n}^{m-1}\left(b_{j}-2 \gamma_{j+1} f\left(V_{j}\right)-2 a_{j}\right) \prod_{i=j}^{m-1} c_{i} .
$$

Now, by (3.2): $E\left[\left\|\xi_{n}\right\| \mid \mathcal{F}_{n-1}\right] \leq \operatorname{tr}(\Sigma)\left\|V_{n}\right\|$.

For $b_{j}$ part,

$$
\begin{aligned}
\sum_{j=n}^{\infty} E\left[b_{j} \mid \mathcal{F}_{j}\right] & =\sum_{j=n}^{\infty} \gamma_{j+1}^{2} E\left[\frac{<\Sigma \xi_{j+1}, \xi_{j+1}>}{\left\|V_{j}\right\|^{2}} \mid \mathcal{F}_{j}\right] \leq \sum_{j=n}^{\infty} \gamma_{j+1}^{2} \frac{\|\Sigma\| E\left[\left\|\xi_{j+1}\right\|^{2} \mid \mathcal{F}_{j}\right]}{\left\|V_{j}\right\|^{2}} \\
& \leq \sum_{j=n}^{\infty} \gamma_{j+1}^{2} \frac{\|\Sigma\| \operatorname{tr}(\Sigma)^{2}\left\|V_{j}\right\|^{2}}{\left\|V_{j}\right\|^{2}}=\sum_{j=n}^{\infty} \gamma_{j+1}^{2} \cdot c
\end{aligned}
$$

thus its rate of convergence is $O\left(\frac{1}{n}\right)$

For $a_{j}$ part, $\sum_{j=n}^{\infty} a_{j}=\sum_{j=n}^{\infty} \gamma_{j+1} Z_{j}, Z_{j}$ is centered and $E\left[Z_{j}^{2}\right]$ is bounded, by lemma 3.1. $E\left[\left|S-S_{n}\right|^{2}\right] \leq \sum_{i>n} E\left[a_{i}^{2}\right]$, whose rate of convergence is $O\left(\frac{1}{n}\right)$, thus $\sum_{j=n}^{\infty} a_{j}$ has the rate of convergence $O\left(\frac{1}{\sqrt{n}}\right)$.

For $c_{j}$ part, by proof of the lemma 3.3. $\prod_{i=n}^{\infty} c_{i}$ has the same convergence properties as $\sum_{i=n}^{\infty} \gamma_{i+1}^{2} \frac{\left\|\xi_{i+1}\right\|^{2}}{\left\|V_{i}\right\|^{2}}$. By $(\underline{3.2})$ :

$$
E\left[\frac{\left\|\xi_{i+1}\right\|^{2}}{\left\|V_{i}\right\|^{2}} \mid \mathcal{F}_{i}\right] \leq E\left[\frac{\operatorname{tr}(\Sigma)^{2}\left\|V_{i}\right\|^{2}}{\left\|V_{i}\right\|^{2}}\right]=\operatorname{tr}(\Sigma)^{2},
$$

thus $\prod_{i=n}^{\infty} c_{i}$ has the rate of convergence $O\left(\frac{1}{n}\right)$.

For $f\left(V_{j}\right)$ part, by assumption 2, rewrite $V_{n}=\sum_{i=1}^{d} a_{i}^{(n)} \theta_{i}$, where $d$ is the dimension. From (3.8), we have: $\sum_{n=1}^{\infty} \gamma_{n+1} f\left(V_{n}\right) \prod_{k=1}^{n-1}\left(1+\gamma_{k+1}^{2} \frac{\left\|\xi_{k+1}\right\|^{2}}{\left\|V_{k}\right\|^{2}}\right)^{-1}<\infty$ with probability 1 . Since we have $\gamma_{n} \asymp_{p} \frac{1}{n}$ and $f\left(V_{n}\right) \geq 0 \forall n$, if $\liminf _{n \rightarrow \infty} f\left(V_{n}\right)=$ $c$, then $\sum_{n=1}^{\infty} \gamma_{n+1} f\left(V_{n}\right) \prod_{k=1}^{n-1}\left(1+\gamma_{k+1}^{2} \frac{\left\|\xi_{k+1}\right\|^{2}}{\left\|V_{k}\right\|^{2}}\right)^{-1}=\infty$, thus $c=0$. 
Now, by nature of eigenvector and eigenvalue, as well as assumption 2: $\theta_{i}^{2}=1$, $\theta_{i} \theta_{j}=0$ for $i \neq j$, and $\left\|V_{n}\right\|^{2}=\sum_{i=1}^{d}\left(a_{i}^{(n)}\right)^{2}$.

Thus:

$$
\begin{aligned}
f\left(V_{n}\right) & =\frac{\left\|\Sigma V_{n}\right\|^{2}}{\left\|V_{n}\right\|^{2}}-\frac{<\Sigma V_{n}, V_{n}>^{2}}{\left\|V_{n}\right\|^{4}} \\
& =\frac{\left(\sum_{i=1}^{d} a_{i}^{(n)} \lambda_{i} \theta_{i}\right)^{2}}{\left\|V_{n}\right\|^{2}}-\mu\left(V_{n}\right)^{2} \\
& =\frac{\sum_{i=1}^{d}\left(a_{i}^{(n)}\right)^{2}\left(\lambda_{i}^{2}-\mu\left(V_{n}\right)^{2}\right)}{\left\|V_{n}\right\|^{2}}
\end{aligned}
$$

which leads to the result: $f\left(V_{j}\right) \rightarrow 0$ with the same rate of $\mu\left(V_{n}\right) \rightarrow \lambda_{1}$.

Thus, $\frac{\leq A_{n} V_{n}, V_{n}>}{\left\|V_{n}\right\|^{2}}$ converges to $\lambda_{1}$ the same rate as $a_{j}$ part, has the rate of convergence $O\left(\frac{1}{\sqrt{n}}\right)$. More precisely, by proof of the Lemma 3.1, $E\left[\left|S_{n+r}-S_{n}\right|^{2}\right] \leq$ $\sum_{i>n} E\left[X_{i}^{2}\right]$ if $\left\{X_{n}\right\}_{n}$ is 0 mean. Then for $a_{j}=\gamma_{j+1} Z_{j}$, we have

$$
E\left[\left|S-S_{n}\right|^{2}\right] \leq \sum_{i>n} E\left[a_{i}^{2}\right] \lesssim_{p} \sum_{i>n} \frac{1}{i^{2}} E\left[Z_{i}^{2}\right] .
$$

Now for $Z_{n}$, by (3.4), we have:

$$
\begin{aligned}
\left\|Z_{n}\right\| & =\| \frac{<\left(A_{n+1}-\Sigma\right) V_{n}, \Sigma V_{n}>}{\left\|V_{n}\right\|^{2}}-\frac{<\left(A_{n+1}-\Sigma\right) V_{n}, V_{n}>}{\left\|V_{n}\right\|^{4}} \cdot\left\langle\Sigma V_{n}, V_{n}>\|\right. \\
& \leq\left\|\frac{<\left(A_{n+1}-\Sigma\right) V_{n}, \Sigma V_{n}>}{\left\|V_{n}\right\|^{2}}\right\|+\left\|\frac{<\left(A_{n+1}-\Sigma\right) V_{n}, V_{n}>}{\left\|V_{n}\right\|^{4}} \cdot<V_{n}, V_{n}>\right\| \\
& \leq \quad\left\|\frac{<\left(A_{n+1}-\Sigma\right) V_{n}, V_{n}>}{\left\|V_{n}\right\|^{2}}\right\| \cdot\|\Sigma\|+\| \frac{<\left(A_{n+1}-\Sigma\right) V_{n}, V_{n}>}{\left\|V_{n}\right\|^{2}} \\
& \quad \cdot \frac{<\Sigma V_{n}, V_{n}>}{\left\|V_{n}\right\|^{2}} \| \\
& \lesssim\left\|\frac{<\left(A_{n+1}-\Sigma\right) V_{n}, V_{n}>}{\left\|V_{n}\right\|^{2}}\right\| \cdot\|\Sigma\| \\
\leq & \left\|A_{n+1}-\Sigma\right\|\|\Sigma\| \\
\leq & \left(\left\|A_{n+1}\right\|+\|\Sigma\|\right)\|\Sigma\| .
\end{aligned}
$$

Thus:

$$
\begin{aligned}
E\left[Z_{n}^{2}\right] & \leq\|\Sigma\|^{2} E\left[\left\|A_{n+1}\right\|^{2}+\|\Sigma\|^{2}+2\left\|A_{n+1}\right\|\|\Sigma\|\right] \\
& \lesssim p=\Sigma \|^{2} E\left[\left\|A_{n+1}\right\|^{2}+\|\Sigma\|^{2}\right] \\
& \asymp_{p}\|\Sigma\|^{2} \cdot\left(E\left[\left\|A_{n}\right\|^{2}\right] \bigvee\|\Sigma\|^{2}\right)
\end{aligned}
$$

So $E\left[\left|S-S_{n}\right|^{2}\right]$ has rate of convergence $O\left(\frac{1}{n} \cdot\|\Sigma\|^{2} \cdot\left(E\left[\left\|A_{n}\right\|^{2}\right] \bigvee\|\Sigma\|^{2}\right)\right)$, thus $\sum_{j=n}^{\infty} a_{j}$ has rate of convergence $O\left(\frac{\|\Sigma\|}{\sqrt{n}} \cdot\left(\sqrt{E\left[\left\|A_{n}\right\|^{2}\right]} \bigvee\|\Sigma\|\right)\right)$.

Lemma 3.6. (1) $V_{n} \rightarrow a_{1}^{(n)} \theta_{1}$ with probability 1 and (2) $\frac{\leq V_{n}, \theta_{1}>^{2}}{\left\|V_{n}\right\|^{2}}$ approach to 1 in the order of $\frac{d\|\Sigma\|}{g \sqrt{n}} \cdot\left(\sqrt{E\left[\left\|A_{n}\right\|^{2}\right]} \bigvee\|\Sigma\|\right)$ with probability 1 .

Proof. (1) We already proved that $f\left(V_{n}\right) \rightarrow 0$ and $\mu\left(V_{n}\right) \rightarrow \lambda_{1}$ in lemma 3.5, thus $\lambda_{i}-\mu\left(V_{n}\right)>0$ for $i \neq 1$ when $n$ large enough. By (3.10), $0=\lim _{n \rightarrow \infty} f\left(V_{n}\right)=$ 
$\lim _{n \rightarrow \infty} \frac{\sum_{i=1}^{d}\left(a_{i}^{(n)}\right)^{2}\left(\lambda_{i}^{2}-\mu\left(V_{n}\right)^{2}\right)}{\left\|V_{n}\right\|^{2}}, a_{i}^{(n)}=0$ when $i \neq 1$, thus $V_{n} \rightarrow a_{1}^{(n)} \theta_{1}$ with probability 1 .

(2) By previous argument, we have:

$$
\begin{aligned}
f\left(V_{n}\right) & =\frac{\sum_{i=1}^{d}\left(a_{i}^{(n)}\right)^{2}\left(\lambda_{i}^{2}-\mu\left(V_{n}\right)^{2}\right)}{\left\|V_{n}\right\|^{2}} \\
& =\frac{\left(a_{1}^{(n)}\right)^{2}\left(\lambda_{1}^{2}-\mu\left(V_{n}\right)^{2}\right)}{\left\|V_{n}\right\|^{2}}+\frac{\sum_{i=2}^{d}\left(a_{i}^{(n)}\right)^{2}\left(\lambda_{i}^{2}-\mu\left(V_{n}\right)^{2}\right)}{\left\|V_{n}\right\|^{2}},
\end{aligned}
$$

convergence with the same rate of $\mu\left(V_{n}\right) \rightarrow \lambda_{1}$, we have $\frac{\sum_{i=2}^{\infty}\left(a_{i}^{(n)}\right)^{2}\left(\lambda_{i}^{2}-\mu\left(V_{n}\right)^{2}\right)}{\left\|V_{n}\right\|^{2}} \rightarrow 0$ at least the same rate as $\frac{\left(a_{1}^{(n)}\right)^{2}\left(\lambda_{1}^{2}-\mu\left(V_{n}\right)^{2}\right)}{\left\|V_{n}\right\|^{2}} \rightarrow 0$.

By part (1), $\mu\left(V_{n}\right)$ has rate of convergence $O\left(\frac{\|\Sigma\|}{\sqrt{n}} \cdot\left(\sqrt{E\left[\left\|A_{n}\right\|^{2}\right]}\right) \bigvee\|\Sigma\|\right)$, we have

$$
\frac{\sum_{i=2}^{\infty}\left(a_{i}^{(n)}\right)^{2}\left(\lambda_{i}^{2}-\lambda_{1}^{2}\right)}{\left\|V_{n}\right\|^{2}} \asymp_{p} \frac{\|\Sigma\|}{\sqrt{n}} \cdot\left(\sqrt{E\left[\left\|A_{n}\right\|^{2}\right]} \bigvee\|\Sigma\|\right) \cdot \frac{\left(a_{1}^{(n)}\right)^{2} \lambda_{1}}{\left\|V_{n}\right\|^{2}},
$$

let $g=\left|\lambda_{1}-\lambda_{2}\right|$, thus:

$$
\begin{aligned}
\sum_{i=2}^{\infty}\left(a_{i}^{(n)}\right)^{2} & \asymp_{p} \quad \frac{\|\Sigma\|}{\sqrt{n}} \cdot\left(\sqrt{E\left[\left\|A_{n}\right\|^{2}\right]} \bigvee\|\Sigma\|\right) \cdot \frac{\left(a_{1}^{(n)}\right)^{2} \lambda_{1}}{\left|\left(\lambda_{i}-\lambda_{1}\right)\left(\lambda_{i}+\lambda_{1}\right)\right|} \\
& \lesssim_{p} \frac{\|\Sigma\|\left\|V_{n}\right\|^{2}}{g \sqrt{n}} \cdot\left(\sqrt{E\left[\left\|A_{n}\right\|^{2}\right]} \bigvee\|\Sigma\|\right)
\end{aligned}
$$

Now by assumption $2,\left\|V_{n}\right\|^{2}=\sum_{i=1}^{d}\left(a_{i}^{(n)}\right)^{2}$, thus:

$$
\left\|V_{n}\right\|^{2}-\left(a_{1}^{(n)}\right)^{2}=\sum_{i=2}^{\infty}\left(a_{i}^{(n)}\right)^{2} \lesssim p \frac{\|\Sigma\|\left\|V_{n}\right\|^{2}}{g \sqrt{n}} \cdot\left(\sqrt{E\left[\left\|A_{n}\right\|^{2}\right]} \bigvee\|\Sigma\|\right) .
$$

Above all:

$$
1-\frac{<V_{n}, \theta_{1}>^{2}}{\left\|V_{n}\right\|^{2}} \lesssim p \frac{\|\Sigma\|}{g \sqrt{n}} \cdot\left(\sqrt{E\left[\left\|A_{n}\right\|^{2}\right]} \bigvee\|\Sigma\|\right)
$$

\section{EXPERIMENT}

The dataset $X \in \mathbb{R}^{10^{6} \times 10}$ was just generated through its singular value decomposition. Specifically, we fix a $10 \times 10$ diagonal matrix $\Sigma=\operatorname{diag}\{1,0.9, \cdots, 0.9\}$ and generate random orthogonal projection matrix $U \in \mathbb{R}^{10^{6} \times 10}$ and random orthogonal matrix $V \in \mathbb{R}^{10 \times 10}$. And the dataset $X=\sqrt{n} U \Sigma V^{T}$, which guarantees that the matrix $A=\frac{1}{n} X^{T} X$ has eigen-gap 0.1. See Figure.1.

\section{Conclusion}

We derived the asymptotic rate of convergence for the estimation of the smallest eigenvalue and corresponding eigenvector of the Krasulina scheme. There are several important questions related to Online PCA.

(1) The Krasulina scheme only requires $O(d)$ storage space complexity against $O\left(d^{2}\right)$ for standard PCA in the offline setting, however, we paid a price in the rate of convergence that is significantly slower than offline setting. See Table.1. 


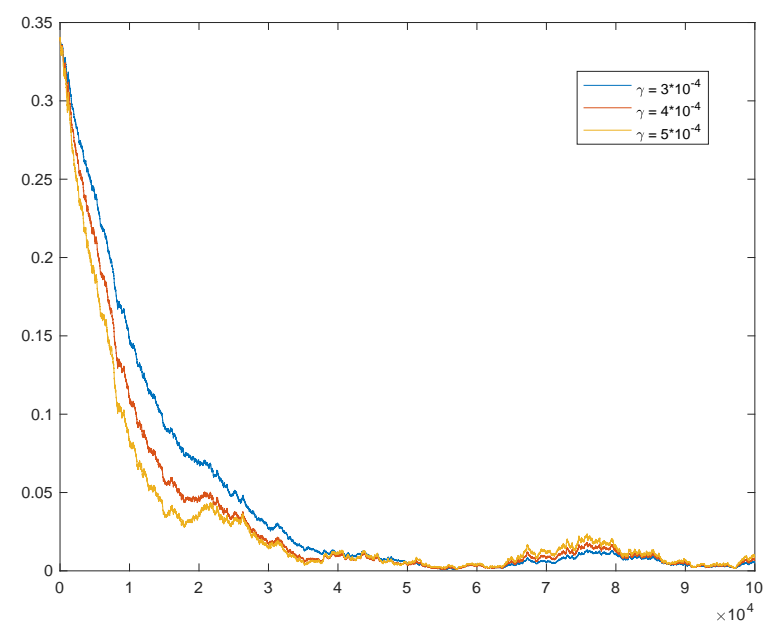

Figure 1. Convergence of Krasulina Scheme

TABle 1. Comparison of different schemes with Gaussian assumption. The convergence rates are given for the operator norm. For the sparse PCA scheme of $\left[\underline{5}, k_{q}^{*}\right.$ denotes the sparsity level of the eigenvectors.

\begin{tabular}{|c|c|c|c|}
\hline Scheme & $\begin{array}{c}\text { Space } \\
\text { complexity }\end{array}$ & Convergence rate & Setting \\
\hline Standard PCA & $O\left(n d^{2}\right)$ & $O\left(\|\Sigma\| \cdot\left(\sqrt{\frac{r(\Sigma)}{n}} \bigvee \frac{r(\Sigma)}{n}\right)\right)$ & Offline \\
\hline Sparse PCA [5] & $O\left(n d^{2}\right)$ & $O\left(\frac{k_{q}^{*}}{n \lambda}\left(d+\log \frac{d}{k_{q}^{*}}\right)\right)$ & Offline \\
\hline Krasulina & $O(d)$ & $O\left(\frac{\|\Sigma\| t r(\Sigma)}{\sqrt{n}}\right)$ & Online \\
\hline
\end{tabular}

An interesting question would be whether the Krasulina scheme can achieve the offline rate of convergence.

The simulation study seems to confirm the slow convergence rate of Krasulina's scheme. It would be interesting to build an acceleration for this scheme. This problem has been investigated by $[6$ where negative numerical results were provided for usual acceleration schemes. Therefore this question remains largely open.

(2) Note that the proof argument in the original paper [11 only gives the consistency of the smallest eigenvalue and corresponding eigenvector for the Krasulina scheme. As we built upon this argument in this paper, we only provide the rate of convergence for the smallest eigenvalue and corresponding eigenvector. We can extend the result to the top eigenvalue and cooresponding eigenvector by (2.1), however, tackling other eigenvalues will require a new argument.

(3) The convergence rates of the Krasulina estimator depends on the multiplier $c$ in the learning rate $\gamma_{n}$ when we take $\gamma_{n}=\frac{c}{n}$. If it is too low, the rate 
of convergence will be slower than $O\left(\frac{1}{\sqrt{n}}\right)$, if it is too high, the constant in the rate of convergence will be large. Is there a simple and practical way to choose $c$ ?

(4) Finally, it would be of interest to derive rates of convergence for other online PCA schemes including Oja and naive PCA.

\section{REFERENCES}

[1] R. Arora, A. Cotter, K. Livescu, and N. Srebro. Stochastic optimization for PCA and PLS. In 2012 50th Annual Allerton Conference on Communication, Control, and Computing (Allerton), pages 861-868, October 2012.

[2] Raman Arora, Andy Cotter, and Nati Srebro. Stochastic optimization of pca with capped msg. In Advances in Neural Information Processing Systems, pages 1815-1823, 2013.

[3] Akshay Balsubramani, Sanjoy Dasgupta, and Yoav Freund. The fast convergence of incremental pca. In Advances in Neural Information Processing Systems, pages 3174-3182, 2013.

[4] Gilles Blanchard, Olivier Bousquet, and Laurent Zwald. Statistical properties of kernel principal component analysis. Machine Learning, 66(2):259-294, 2007.

[5] T. Tony Cai, Zongming Ma, and Yihong Wu. Sparse PCA: Optimal rates and adaptive estimation. The Annals of Statistics, 41(6):3074-3110, December 2013. arXiv: 1211.1309.

[6] Christopher De Sa, Bryan He, Ioannis Mitliagkas, Christopher R, and Peng Xu. Accelerated stochastic power iteration. arXiv preprint arXiv:1707.02670, 2017.

[7] Prateek Jain, Chi Jin, Sham M Kakade, Praneeth Netrapalli, and Aaron Sidford. Streaming pca: Matching matrix bernstein and near-optimal finite sample guarantees for ojas algorithm. In Conference on Learning Theory, pages 1147-1164, 2016.

[8] Vladimir Koltchinskii and Karim Lounici. Concentration Inequalities and Moment Bounds for Sample Covariance Operators. arXiv:1405.2468 [math], May 2014. arXiv: 1405.2468.

[9] Vladimir Koltchinskii, Karim Lounici, et al. Asymptotics and concentration bounds for bilinear forms of spectral projectors of sample covariance. In Annales de l'Institut Henri Poincaré, Probabilités et Statistiques, volume 52, pages 1976-2013. Institut Henri Poincaré, 2016.

[10] Vladimir Koltchinskii, Karim Lounici, et al. Normal approximation and concentration of spectral projectors of sample covariance. The Annals of Statistics, 45(1):121-157, 2017.

[11] T. P. Krasulina. The method of stochastic approximation for the determination of the least eigenvalue of a symmetrical matrix. USSR Computational Mathematics and Mathematical Physics, 9(6):189-195, 1969.

[12] Ioannis Mitliagkas, Constantine Caramanis, and Prateek Jain. Memory limited, streaming pca. In Advances in Neural Information Processing Systems, pages 2886-2894, 2013.

[13] Erkki Oja and Juha Karhunen. On stochastic approximation of the eigenvectors and eigenvalues of the expectation of a random matrix. Journal of mathematical analysis and applications, 106(1):69-84, 1985.

[14] Sam T Roweis. Em algorithms for pca and spca. In Advances in neural information processing systems, pages 626-632, 1998.

[15] Ohad Shamir. A stochastic pca and svd algorithm with an exponential convergence rate. In International Conference on Machine Learning, pages 144-152, 2015.

[16] Vincent Q. Vu and Jing Lei. Minimax rates of estimation for sparse PCA in high dimensions. In International Conference on Artificial Intelligence and Statistics, pages 1278-1286, 2012.

[17] Manfred K Warmuth and Dima Kuzmin. Randomized pca algorithms with regret bounds that are logarithmic in the dimension. In Advances in neural information processing systems, pages 1481-1488, 2007.

[18] Juyang Weng, Yilu Zhang, and Wey-Shiuan Hwang. Candid covariance-free incremental principal component analysis. IEEE Transactions on Pattern Analysis and Machine Intelligence, 25(8):1034-1040, 2003.

[19] Laurent Zwald and Gilles Blanchard. On the convergence of eigenspaces in kernel principal component analysis. In Advances in neural information processing systems, pages 1649-1656, 2006 .

School of Mathematics, Georgia Institute of Technology, Altanta, GA 30313

E-mail address: jchen444@math.gatech.edu 